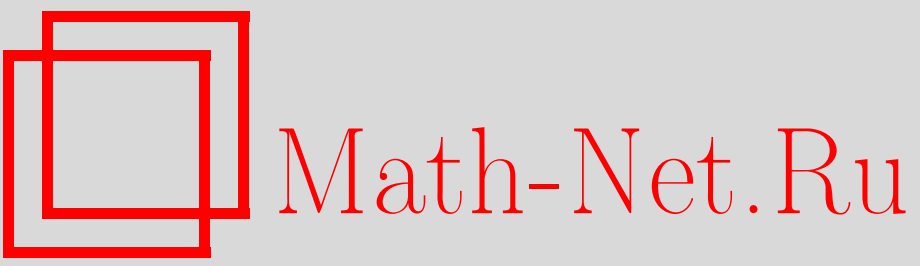

М. Бертола, Б. Эйнард, Дж. Харнад, Дуальность спектральных кривых, возникающая в двуматричных моделях, ТМФ, 2003, том 134, номер 1, 32-45

DOI: https://doi.org/10.4213/tmf138

Использование Общероссийского математического портала Math-Net.Ru подразумевает, что вы прочитали и согласны с пользовательским соглашением

http://www . mathnet.ru/rus/agreement

Параметры загрузки:

IP : 54.224 .187 .69

26 апреля 2023 г., 16:09:15 


\section{ДУАЛЬНОСТЬ СПЕКТРАЛЬНЫХ КРИВЫХ, ВОЗНИКАЮЩАЯ В ДВУМАТРИЧНЫХ МОДЕЛЯХ}

Рассматривается двуматричная модель, мера в которой задается экспонентой суммы многочленов от двух различных переменных. Показано, как можно вывести последовательность пар "дуальных" конечномерных систем ОДУ для соответствующих биортогональных многочленов. Доказана обратная теорема, утверждающая, что можно восстановить указанные меры из пар полубесконечных конечнозонных матриц, которые задают соотношения рекурсии и удовлетворяют струнному уравнению. В пределе $N \rightarrow \infty$ доказано, что полученные дуальные системы имеют одну и ту же спектральную кривую.

Ключевые слова: модель случайных матриц, асимптотический анализ, дуальность ОДУ.

\section{1. ВВЕДЕНИЕ}

Рассматривается двуматричная модель [1]-[5], в которой описываются ансамбли, состоящие из пар эрмитовых матриц $M_{1}$ и $M_{2}$ размера $N \times N$ с $U(N)$-инвариантной вероятностной мерой вида

$$
\frac{1}{\tau_{N}} d \mu\left(M_{1}, M_{2}\right):=\frac{1}{\tau_{N}} e^{K \operatorname{tr}\left(-V_{1}\left(M_{1}\right)-V_{2}\left(M_{2}\right)+M_{1} M_{2}\right)} d M_{1} d M_{2} .
$$

Здесь $d M_{1} d M_{2}$ - стандартная мера Лебега для пар эрмитовых матриц, а функции $V_{1}$ и $V_{2}$ суть многочлены степеней $d_{1}+1$ и $d_{2}+1$, соответственно. Величины $V_{1}$ и $V_{2}$ в дальнейшем называются потенциалами. Считается, что общий положительньй масштабный множитель $K$ в экспоненте пропорционален $N$ в пределе больших $N$. Также предполагается, что оба потенциала вешественны и ограничены снизу (для обеспечения сходимости).

Известно, что нормировочный множитель (статистическая сумма)

$$
\tau_{N}=\int_{M_{1}} \int_{M_{2}} d \mu
$$

\footnotetext{
* Centre de Recherches Mathématiques, Université de Montréal, Montréal, Canada. E-mail: bertola@crm.umontreal.ca; harnad@crm.umontreal.ca

${ }^{\dagger}$ Department of Mathematics and Statistics, Concordia University, Montréal, Canada

${ }^{\ddagger}$ Service de Physique Théorique, CEA, Saclay, France. E-mail: eynard@spht.saclay.cea.fr
} 
представляет собой тау-функцию иерархии КП относительно каждого из наборов параметров деформации (коэффициенты двух многочленов $V_{1}, V_{2}$ ) и одновременно задает решения уравнений двумеризованной цепочки Тоды [6], [7]. Ключевым объектом теории являются корреляционные функции для собственных значений этих двух матриц. Как и в одноматричном случае, эти корреляционные функции можно найти в терминах некоторых ядер интегральных операторов Фредгольма. Напомним, что в одноматричной модели с мерой

$$
\frac{1}{\tau_{N}} d \mu(M):=\frac{1}{\tau_{N}} e^{\operatorname{tr}(-V(M))} d M
$$

вычисление таких ядер сводится к построению системы ортогональных многочленов $P_{n}(x)$ для пространства $L^{2}\left(\mathbb{R}, e^{-V(x)} d x\right)$. В терминах этих многочленов ядро имеет вид

$$
\stackrel{N}{K}\left(x, x^{\prime}\right)=\sum_{n=0}^{N-1} P_{n}(x) e^{-V(x) / 2} P_{n}\left(x^{\prime}\right) e^{-V\left(x^{\prime}\right) / 2} .
$$

Заметим, что в двуматричных моделях необходимо вычислить четыре соответствующих ядра, чтобы получить статистические корреляции собственных значений. Для $m$-матричных моделей надо вычислить $m^{2}$ таких ядерных операторов.

В двуматричной модели эти ядра выражаются в терминах подходящим образом определенных последовательностей биортогональных многочленов. Под такими последовательностями подразумеваются две последовательности многочленов от одной переменной

$$
\pi_{n}(x)=x^{n}+\cdots, \quad \sigma_{n}(y)=y^{n}+\cdots, \quad n=0,1, \ldots,
$$

которые ортогональны относительно связанной меры на пространстве произведений:

$$
\int_{\mathbb{R}} \int_{\mathbb{R}} d x d y \pi_{n}(x) \sigma_{m}(y) e^{-K V_{1}(x)-K V_{2}(y)+K x y}=h_{n} \delta_{m n},
$$

где $V_{1}(x)$ и $V_{2}(y)$ - многочлены, входящие в меру интегрирования для двуматричной модели (1). Соотношения ортогональности однозначно определяют оба семейства многочленов, если только такие семейства существуют [2]. Четыре соответствующих ядра выражаются следуюшим образом через эти биортогональные многочлены:

$$
\begin{aligned}
& \stackrel{N}{K}_{12}(x, y)=\sum_{n=0}^{N-1} \frac{1}{h_{n}} \pi_{n}(x) \sigma_{n}(y) e^{-K V_{1}(x)} e^{-K V_{2}(y)}, \\
& \stackrel{N}{K}_{11}\left(x, x^{\prime}\right)=\int_{\mathbb{R}} d y \stackrel{N}{K}_{12}(x, y) e^{K x^{\prime} y}, \\
& \stackrel{N}{K}_{22}\left(y^{\prime}, y\right)=\int_{\mathbb{R}} d x \stackrel{N}{K_{12}}(x, y) e^{K x y^{\prime}}, \\
& \stackrel{N}{K}_{21}\left(y^{\prime}, x^{\prime}\right)=\int_{\mathbb{R}} \int_{\mathbb{R}} d x d y \stackrel{N}{K_{12}}(x, y) e^{K x y^{\prime}} e^{K x^{\prime} y} .
\end{aligned}
$$

Все статистические свойства спектров такого двуматричного ансамбля могут быть при этом выражены в терминах этих ядер [5] и соответствуюших интегральных операторов

2 Теоретическая и математическая физика, т. 134, № 1, 2003 г. 
Фредгольма $\stackrel{N}{K}{ }_{i j}, i, j=1,2$. Например, плотность собственных значений первой матрицы имеет вид

$$
\stackrel{N}{\rho}_{1}(x)=\frac{1}{N} \stackrel{N}{K}_{11}(x, x),
$$

в то время как корреляционная функция двух собственных значений первой матришы равна

$$
\stackrel{N}{\rho}_{11}\left(x, x^{\prime}\right)=\frac{1}{N^{2}}\left(\stackrel{N}{K}_{11}(x, x) \stackrel{N}{K}{ }_{11}\left(x^{\prime}, x^{\prime}\right)-\stackrel{N}{K}_{11}\left(x, x^{\prime}\right) \stackrel{N}{K}{ }_{11}\left(x^{\prime}, x\right)\right),
$$

а корреляционная функция двух собственных значений, одно из которых относится к первой, а другое - ко второй матрице, равна

$$
\stackrel{N}{\rho}_{12}(x, y)=\frac{1}{N^{2}}\left(\stackrel{N}{K}_{11}(x, x) \stackrel{N}{K}{ }_{22}(y, y)-\stackrel{N}{K}_{12}(x, y)\left(\stackrel{N}{K}_{21}(y, x)-e^{K x y}\right)\right) .
$$

Любые другие корреляционные функции для $m$ собственных значений могут быть записаны схожим образом с помошью детерминантов конечномерных матрищ, матричные элементы которых суть эти четыре ядра.

Основная задача данной работы состоит в том, чтобы вывести и проанализировать некоторые системы ОДУ, которым удовлетворяют квазимногочлены $\psi_{n}(x):=\pi_{n}(x) \times$ $e^{-V_{1}(x)}, \phi_{n}(y):=\sigma_{n}(y) e^{-V_{2}(y)}$ и их образы Фурье-Лапласа. В разделе 2 собраны основные результаты для случая конечномерных $(N \times N)$-матрищ (детали и доказательства см. в работах [8], [9]). В разделе 3 соответствуюшие результаты выводятся в пределе $N \rightarrow \infty$ достаточно простым образом.

Подробнее доказательство предложения 1 и неабелева версия рассуждения о поперечности (см. раздел 3 ) описаны в нашей совместной работе с Хуртубисом [9].

\section{2. СКЛАДЫВАНИЕ И ДУАЛЬНОСТЬ В СИСТЕМАХ ОДУ}

Рассматривая нормированные квазимногочлены

$$
\psi_{n}(x)=\frac{1}{\sqrt{h_{n}}} \pi_{n}(x) e^{-K V_{1}(x)}, \quad \phi_{n}(y)=\frac{1}{\sqrt{h_{n}}} \sigma_{n}(y) e^{-K V_{2}(y)}, \quad n=0, \ldots, \infty,
$$

как компоненты пары полубесконечных вектор-столбцов

$$
\underset{\infty}{\Psi}=\left(\psi_{0}, \psi_{1}, \ldots, \psi_{n}, \ldots\right)^{\mathrm{t}}, \quad \underset{\infty}{\Phi}=\left(\phi_{0}, \phi_{1}, \ldots, \phi_{n}, \ldots\right)^{\mathrm{t}}
$$

получим пары полубесконечных матриц $Q$ и $P$, которые описывают соответственно умножение $\underset{\infty}{\Psi}$ на $x$ и взятие производной $-(1 / K) d / d x$. Аналогично можно построить транспонированные матришы $Q^{\mathrm{t}}$ и $P^{\mathrm{t}}$, применяя операторы дифференширования $-(1 / K) d / d y$ или умножения на $-y$ к вектору $\underset{\infty}{\Phi}$. По построению эти матрицы удовлетворяют коммутационным соотношениям Гейзенберга (или “струнному уравнению")

$$
[P, Q]=\frac{1}{K} \mathbf{1} .
$$


Помимо этих квазимногочленов потребуются также их образы Фурье-Лапласа и соответствующие полубесконечные вектор-строки

$$
\underline{\Psi}(y):=\left(\underline{\psi}_{0}, \ldots, \underline{\psi}_{n}, \ldots\right), \quad \underline{\Phi}(x):=\left(\underline{\phi}_{0}, \ldots, \underline{\phi}_{n}, \ldots\right)
$$

c компонентами

$$
\underline{\psi}_{n}(y):=\int_{\mathbb{R}} d x e^{K x y} \psi_{n}(x), \quad \underline{\phi}_{n}(x):=\int_{\mathbb{R}} d y e^{K x y} \phi_{n}(y) .
$$

С помощью интегрирования по частям можно найти рекуррентные соотношения для этих величин по отношению к умножению и взятию производной:

$$
\begin{array}{ll}
x \underline{\Phi}(x)=\frac{\Phi}{\infty}(x) Q, & \frac{1}{K} \frac{d}{d x} \frac{\Phi}{\infty}(x)=\frac{\Phi}{\infty}(x) P, \\
y \frac{\Psi}{\infty}(y)=\frac{\Psi}{\infty}(y) Q^{\mathrm{t}}, & \frac{1}{K} \frac{d}{d y} \frac{\Psi}{\infty}(y)=\frac{\Psi}{\infty}(y) P^{\mathrm{t}} .
\end{array}
$$

Также из интегрирования по частям следует [8], что матрицы $P$ и $Q$ обладают конечнозонной структурой:

$$
\begin{aligned}
& Q:=\left[\begin{array}{ccccc}
\alpha_{0}(0) & \gamma(0) & 0 & 0 & \ldots \\
\alpha_{1}(1) & \alpha_{0}(1) & \gamma(1) & 0 & \ldots \\
\vdots & \ddots & \ddots & \ddots & \ddots \\
\alpha_{d_{2}}\left(d_{2}\right) & \ldots & \alpha_{0}\left(d_{2}\right) & \gamma\left(d_{2}\right) & \ddots \\
\ldots \ldots & \ldots & \ldots & \ldots \\
0 & \ddots & \ddots & \ddots & \ddots
\end{array}\right], \\
& P:=\left[\begin{array}{ccccccc}
\beta_{0}(0) & \beta_{1}(1) & \ldots & \ldots & \beta_{d_{1}}\left(d_{1}\right) & \ldots & \ldots \\
\gamma(0) & \beta_{0}(1) & \beta_{1}(2) & \ddots & \ddots & \beta_{d_{1}}\left(d_{1}+1\right) & \ldots \\
0 & \gamma(1) & \beta_{0}(2) & \ddots & \ddots & \ddots & \ddots \\
0 & 0 & \gamma(2) & \beta_{0}(3) & \ddots & \ddots & \ddots \\
\ldots \ldots & \ldots & \ldots \ldots \ldots \ldots \ldots \ldots \ldots \ldots \ldots \ldots \\
0 & \ddots & \ddots & \ddots & \ddots & \ddots & \ddots
\end{array}\right],
\end{aligned}
$$

где $\gamma(n) \neq 0$ для всех $n \in \mathbb{N}$. Эта структура по существу следует из того, что матрицы $\left(P-V_{1}^{\prime}(Q)\right),\left(Q-V_{2}^{\prime}(P)\right)$ соответственно строго нижне- и верхнетреугольны. В самом деле, в базисе квазимногочленов очевидно, что

$$
\begin{aligned}
\sum_{m=0}^{\infty}\left(P-V_{1}^{\prime}(Q)\right)_{n m} \psi_{m}(x) & =\left(-\frac{1}{K} \frac{d}{d x}-V_{1}^{\prime}(x)\right) \psi_{n}(x)= \\
& =c \psi_{n-1}(x)+\text { квазимногочлены степени меньше } n
\end{aligned}
$$

и что матрицы $Q$ и $P^{\mathrm{t}}$, задающие соответственно умножения на $x$ и $y$, не могут иметь более одной ненулевой диагонали над главной диагональю. Обратное утверждение также выполняется, что будет детально обсуждаться ниже. 
ПРЕДЛОЖЕНИЕ 1. Предположим, что матрищъ $P$ и $Q$ обладают вышеприведенной зонной структурой и что выссшая диагональ матрицы $Q$ и низиая диагональ матрицы $P$ содержат ненулевые әлементы. Тогда два следующих условия эквивалентны:

1) коммутатор $[P, Q]$ представляет собой диагональную матричу;

2) существуют два многочлена соответствующих степеней $d_{1} u d_{2}$, которье обозначаются $V_{1}^{\prime}(x)$ u $V_{2}^{\prime}(y)$, такие, что

$$
\left(P-V_{1}^{\prime}(Q)\right)_{\geqslant 0}=0, \quad\left(Q-V_{2}^{\prime}(P)\right)_{\leqslant 0}=0,
$$

где нижние индексы $\leqslant 0$ или $\geqslant 0$ обозначают соответственно нижнюю или верхнюю части матрииъ.

ДокАЗАТЕльСтво.Детальное доказательство этого утверждения содержится в работе [9]. Здесь же только отметим, что если заданы зонные структуры двух полубесконечных матриц $P$ и $Q$, то многочлен $V_{1}^{\prime}(x)$ однозначно определяется соотношением

$$
\left(P-V_{1}^{\prime}(Q)\right) e_{0}=0, \quad e_{0}:=(1,0,0,0, \ldots)^{\mathrm{t}},
$$

и само его сушествование обеспечивается условием $\gamma(n) \neq 0$. Сходным образом многочлен $V_{2}^{\prime}(y)$ также оказывается однозначно определенным. Тогда можно показать, что все соотношения, содержашиеся в (3), выполнены для этих многочленов. Обратно, если сушествуют два многочлена $V_{1}^{\prime}$ и $V_{2}^{\prime}$, удовлетворяюшие уравнениям (3), то

$$
\left[P, Q-V_{2}(P)\right]=[P, Q]=\left[P-V_{1}(Q), Q\right] .
$$

Но при этом левая часть этого соотношения нестрого верхнетреугольна, а правая часть нестрого нижнетреугольна, так что матриша $[P, Q]$ должна быть диагональна.

Из структуры (2) матриц $P$ и $Q$ следует, что четыре последовательности $\psi_{n}, \underline{\psi}_{n}$, $\phi_{n}$ и $\underline{\phi}_{n}$ удовлетворяют соотношениям рекурсии как по операции умножения, так и по операции взятия производной:

$$
\begin{aligned}
x \psi_{n} & =\gamma(n) \psi_{n+1}+\sum_{j=0}^{d_{2}} \alpha_{j}(n) \psi_{n-j}, \\
-\frac{1}{K} \frac{d}{d x} \psi_{n} & =\gamma(n-1) \psi_{n-1}+\sum_{j=0}^{d_{1}} \beta_{j}(n+j) \psi_{n+j} ; \\
y \phi_{n} & =\gamma(n) \phi_{n+1}+\sum_{j=0}^{d_{1}} \beta_{j}(n) \phi_{n-j} \\
-\frac{1}{K} \frac{d}{d x} \phi_{n} & =\gamma(n-1) \phi_{n-1}+\sum_{j=0}^{d_{2}} \alpha_{j}(n+j) \phi_{n+j} .
\end{aligned}
$$


Из конечных рекуррентных соотношений, выполненных для квазимногочленов $\psi_{n}(x)$ и $\phi_{n}(y)$, следует набор обобщенных соотношений Кристоффеля-Дарбу [4], [10], из которых следует, что ядра $\stackrel{N}{K}{ }_{11}\left(x, x^{\prime}\right)$ и $\stackrel{N}{K_{22}}\left(y^{\prime}, y\right)$ могут быть представлены в виде

$$
\begin{aligned}
& \stackrel{N}{K}_{11}\left(x, x^{\prime}\right)=\frac{\left(\underline{\underline{\Phi}}^{N-1}\left(x^{\prime}\right), \stackrel{N}{\mathbb{A}} \underset{N}{\Psi}(x)\right)}{x^{\prime}-x}, \\
& \mathbb{A}:=\left[\begin{array}{ccccc|c}
0 & 0 & \ldots & 0 & 0 & -\gamma(N-1) \\
\hline \alpha_{d_{2}}(N) & \ldots & \ldots & \alpha_{2}(N) & \alpha_{1}(N) & 0 \\
0 & \alpha_{d_{2}}(N+1) & \ldots & \ldots & \alpha_{1}(N+1) & 0 \\
0 & 0 & \alpha_{d_{2}}(N+2) & \ldots & \ldots & 0 \\
\vdots & \vdots & \vdots & \vdots & \vdots & \vdots \\
0 & 0 & 0 & \ldots & \alpha_{d_{2}}\left(N+d_{2}-1\right) & 0
\end{array}\right],
\end{aligned}
$$

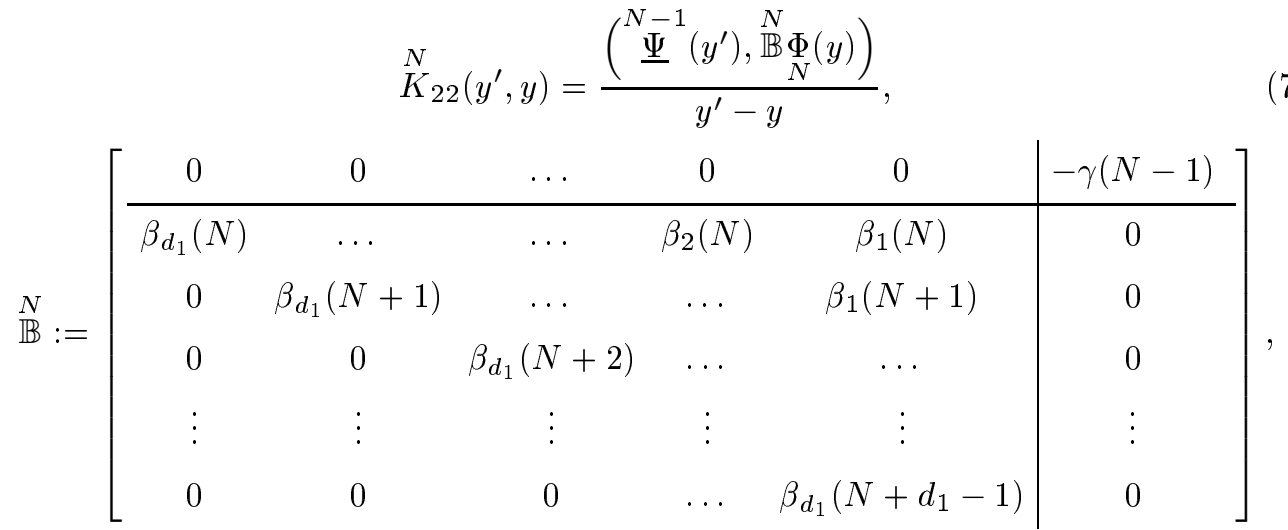

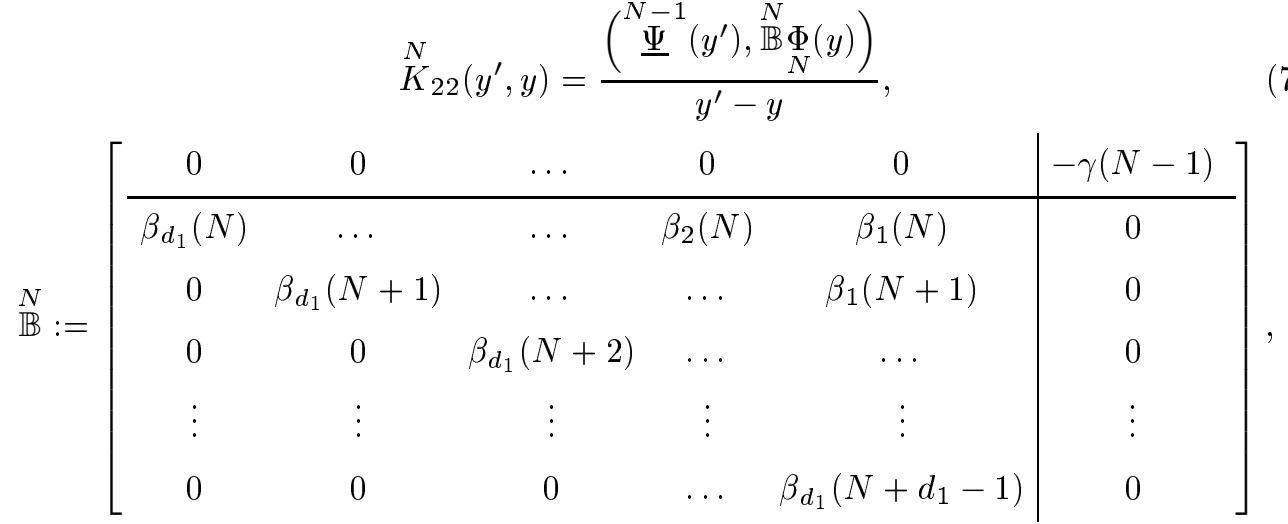

где $\underset{N}{\Psi}(x), \underset{N}{\Phi}(y), \stackrel{N-1}{\Psi}(y)$ и $\stackrel{N-1}{\Phi}(x)$ суть вектор-столбцы или вектор-строки размерностей $d_{1}+1$ и $d_{2}+1$, заданные соотношениями

$$
\begin{aligned}
\Psi_{N}(x) & =\left[\psi_{N-d_{2}}, \ldots, \psi_{N}\right]^{\mathrm{t}}, & \underline{\Phi}_{N}(y) & =\left[\phi_{N-d_{1}}, \ldots, \phi_{N}\right]^{\mathrm{t}}, \\
\underline{\Psi}^{N-1}(y) & =\left[\underline{\psi}_{N-1}, \ldots, \underline{\psi}_{N+d_{1}-1}\right], & \underline{\Phi}^{N-1}(x) & =\left[\underline{\phi}_{N-1}, \ldots, \underline{\phi}_{N+d_{2}-1}\right] .
\end{aligned}
$$

Матрицы $\mathbb{A}(6), \stackrel{N}{\mathbb{B}}(7)$ задают два спаривания (которые мы будем назьвать спариваниями Кристоффеля-Дарбу) между векторами $\underset{N}{\Psi}$ и $\underline{\Phi}^{N-1}$ и между векторами $\underset{N}{\Phi} \stackrel{N-1}{\underline{\Psi}}$. Мы называем эти пары дуальнымми окнами.

Ключевое наблюдение состоит в том, что всякий квазимногочлен $\psi_{j}(x)$ может быть однозначным образом представлен для любого заданного $N \geqslant d_{2}$ через линейные комбинации любых $d_{2}+1$ последовательных базисных элементов $\psi_{N-d_{2}}, \ldots, \psi_{N} c$ nолиномиальными коэффиииентами. Эта процедура называется складьванием (folding) 
пространства в окно (window), представляюшее собой линейную оболочку множества векторов $\underset{N}{\mathbf{\Psi}}=\left[\psi_{N-d_{2}}, \ldots, \psi_{N}\right]^{\mathrm{t}}$. Это складьвание достигается с помощью соотношений $x$-рекурсии для квазимногочленов из соотношения (4), которые позволяют выразить $(N+1)$-й квазимногочлен через $d_{2}+1$ предшествуюших, но с коэффициентами, которые представляют собой многочлены относительно переменной $x$. Итерируя эту процедуру, можно получить складывание.

Все вышесказанное можно выразить в матричном виде:

$$
\begin{aligned}
\underset{N}{\mathbf{a}}(x) \underset{N}{\Psi}(x) & :=\left[\begin{array}{cccccc}
0 & 1 & 0 & \ldots & \ldots & 0 \\
0 & 0 & \ddots & \ddots & \ddots & 0 \\
\ldots \ldots \ldots \ldots \ldots \ldots \ldots \ldots \ldots \ldots \ldots \ldots \ldots \\
0 & 0 & 0 & \ddots & \ddots & 1 \\
\frac{-\alpha_{d_{2}(N)}}{\gamma(N)} & \ldots & \ldots & \ldots & \frac{-\alpha_{1}(N)}{\gamma(N)} & \frac{x-\alpha_{0}(N)}{\gamma(N)}
\end{array}\right]\left[\begin{array}{c}
\psi_{N-d_{2}} \\
\vdots \\
\vdots \\
\psi_{N}
\end{array}\right]= \\
= & {\left[\begin{array}{c}
\psi_{N-d_{2}+1} \\
\vdots \\
\vdots \\
\psi_{N+1}
\end{array}\right]=\underset{N+1}{\Psi}(x), \quad N \geqslant d_{1} . }
\end{aligned}
$$

Матрица $\underset{N}{\mathbf{a}}$ обратима, поскольку ее определитель равен $\alpha_{d_{2}}(n) / \gamma(n)$, а величина $\alpha_{d_{2}}(n)$ не обрашается в нуль вследствие соотношения $\left(Q-V_{2}^{\prime}(P)\right)_{\leqslant 0}=0$. Эту матрицу в дальнейшем будем называть лестничной матрицей. Полностью аналогичное соотношение верно и для квазимногочленов $\phi_{n}(y)$ (см. ниже (13)) и для соответствуюших образов Фурье-Лапласа.

С помощью такого складывания можно также выразить действие любого оператора, имеюшего конечный размер в терминах зон, как $\left(d_{2}+1\right) \times\left(d_{2}+1\right)$-матричного многочлена от переменной $x$. Наиболее важный случай - это складьвание оператора $P=-(1 / K) d / d x$. Получим

$$
\begin{aligned}
- & \frac{1}{K} \frac{d}{d x} \underset{N}{\Psi}=\underset{N}{\underset{N}{\Psi}}=\stackrel{N}{D_{1}}(x) \underset{N}{\Psi}:= \\
& \left.:=(\underset{\gamma}{\gamma} \underset{N-1}{\mathbf{a}}(x))^{-1}+\stackrel{N}{\beta_{0}}+\sum_{j=1}^{d_{1}}{\stackrel{N}{\beta_{j}}}_{N+j-1}^{\mathbf{a}}(x) \underset{N+j-2}{\mathbf{a}}(x) \ldots \underset{N}{\mathbf{a}}(x)\right) \underset{N}{\Psi}
\end{aligned}
$$

где

$$
\begin{aligned}
\stackrel{N}{\beta}_{j} & :=\operatorname{diag}\left[\beta_{j}\left(N+j-d_{2}\right), \beta_{j}\left(N+j-d_{2}+1\right), \ldots, \beta_{j}(N+j)\right], \quad j=0, \ldots, d_{1}, \\
\stackrel{N}{\gamma} & :=\operatorname{diag}\left[\gamma\left(N-1-d_{2}\right), \gamma\left(N-d_{2}\right), \ldots, \gamma\left(N+d_{2}-1\right)\right] .
\end{aligned}
$$

Соответствуюшее утверждение для многочленов $\phi_{n}$ получается при перестановке местами $x$ и $y, \psi_{n}$ и $\phi_{n}, d_{1}$ и $d_{2}, \alpha_{j}$ и $\beta_{j}$ и т.д. При этом получается аналогичным 
образом определенная матрища $D_{2}(y)$, задающая действие производной на квазимногочленах $\phi_{n}$. Используя обозначения

$$
\begin{aligned}
& \stackrel{N}{\alpha_{j}}:=\operatorname{diag}\left[\alpha_{j}\left(N+j-d_{1}\right), \alpha_{j}\left(N+j-d_{1}+1\right), \ldots, \alpha_{j}(N+j)\right], \quad j=0, \ldots d_{2}, \\
& \stackrel{N}{\gamma}:=\operatorname{diag}\left[\gamma\left(N-d_{1}-1\right), \ldots, \gamma(N-1)\right] \text {, } \\
& \underset{N}{\mathbf{b}}(y):=\left[\begin{array}{ccccccc}
0 & 1 & 0 & \ldots & \ldots & \ldots & 0 \\
0 & 0 & \ddots & \ddots & \ddots & \ddots & 0 \\
\ldots \ldots & \ldots & \ldots & \ldots \ldots & \ldots & \ldots \ldots & \ldots \ldots \\
0 & 0 & 0 & \ddots & \ddots & \ddots & 1 \\
\frac{-\beta_{d_{1}(N)}(N)}{\gamma(N)} & \ldots & \ldots & \ldots & \ldots & \frac{-\beta_{1}(N)}{\gamma(N)} & \frac{y-\beta_{0}(N)}{\gamma(N)}
\end{array}\right], \quad N \geqslant d_{1},
\end{aligned}
$$

получим

$$
\begin{gathered}
\underset{N}{\mathbf{b}}(y) \underset{N}{\Phi}(y)=\underset{N+1}{\Phi}(y), \\
-\frac{1}{K} \frac{d}{d y} \underset{N}{\Phi}=Q^{\mathrm{t}} \underset{N}{\Phi}=\stackrel{N}{D}_{2}(y) \underset{N}{\Phi}:=\left(\underset{\gamma}{\gamma}(\underset{N-1}{\mathbf{b}}(y))^{-1}+\stackrel{\alpha}{\alpha}^{N}+\right. \\
\left.+\sum_{j=1}^{d_{2}} \stackrel{N}{\alpha_{j}} \underset{N+j-1}{\mathbf{b}}(y) \underset{N+j-2}{\mathbf{b}}(y) \ldots \underset{N}{\mathbf{b}}(y)\right) \underset{N}{\Phi}
\end{gathered}
$$

Можно повторить сходную процедуру и для соответствующих образов Фурье-Лапласа. При этом необходимые определения и соотношения даются следующими формулами:

$$
\begin{aligned}
& \underset{\mathbf{a}}{N}(x):=\left[\begin{array}{ccccc}
\frac{x-\alpha_{0}(N)}{\gamma(N-1)} & 1 & 0 & \ldots & 0 \\
\frac{-\alpha_{1}(N+1)}{\gamma(N-1)} & 0 & 1 & \ddots & 0 \\
\ldots \ldots \ldots \ldots & \ldots \ldots & \ldots \ldots \\
\vdots & 0 & \ddots & 0 & 1 \\
\frac{-\alpha_{d_{2}}\left(N+d_{2}\right)}{\gamma(N-1)} & 0 & \ldots & 0 & 0
\end{array}\right] \in g l_{d_{2}+1}[x] \\
& \begin{array}{c}
\underline{\mathbf{b}}(y):=\left(\begin{array}{ccccc}
\frac{y-\beta_{0}(N)}{\gamma(N-1)} & 1 & 0 & \ldots & 0 \\
\frac{-\beta_{1}(N+1)}{\gamma(N-1)} & 0 & 1 & \ddots & 0 \\
\ldots \ldots \ldots \ldots & \ldots & \ldots & \ldots \\
\vdots & 0 & \ddots & 0 & 1 \\
\frac{-\beta_{d_{1}}\left(N+d_{1}\right)}{\gamma(N-1)} & 0 & \ldots & 0 & 0
\end{array}\right) \in g l_{d_{1}+1}[y], \\
\underline{\Psi}^{N-1}=\underline{\Psi} \underline{\mathbf{a}}(x), \quad \underline{\Phi}=\underline{\Phi} \underline{\mathbf{b}}(y),
\end{array} \\
& \frac{1}{K} \frac{d}{d y} \stackrel{N-1}{\underline{\Psi}}(y)=\underline{\underline{\Psi}}^{N-1}(y) \underline{N}_{2}(y), \quad N \geqslant d_{1}+1, \\
& \frac{1}{K} \frac{d}{d x} \stackrel{N-1}{\Phi}(x)=\underline{\Phi}^{N-1}(x) \underline{D}_{1}(x), \quad N \geqslant d_{2}+1,
\end{aligned}
$$


где

$$
\begin{aligned}
\underline{D}_{2}(y) & :=\left(\begin{array}{l}
N \\
\underline{\mathbf{b}}
\end{array}\right)^{-1} \underline{\underline{\gamma}}^{N-1}+\underline{\underline{\alpha}}_{0}^{N-1}+\sum_{j=1}^{d_{2}} \underline{\underline{\mathbf{b}}} \underline{\mathbf{b}} \ldots \underline{\mathbf{b}}^{N-1} \underline{\alpha}_{j}, \\
\underline{D}_{1}(x) & :=\left(\begin{array}{l}
N \\
\underline{\mathbf{a}}
\end{array}{ }^{-1} \underline{\gamma}^{N-1}+\underline{\beta}_{0}^{N-1}+\sum_{j=1}^{d_{1}} \underline{\mathbf{a}}^{N-1 N-2} \underline{\mathbf{a}}^{N-\underline{\mathbf{a}}^{N}} \underline{\beta}_{j},\right. \\
\underline{N}_{j} & :=\operatorname{diag}\left(\alpha_{j}(N-1), \ldots, \alpha_{j}\left(N+d_{1}-1\right)\right), \\
\underline{\underline{\alpha}}_{j} & \underline{N-1}_{j}:=\operatorname{diag}\left(\beta_{j}(N-1), \ldots, \beta_{j}\left(N+d_{2}-1\right)\right) .
\end{aligned}
$$

Подводя итог, мы получим четыре последовательности систем линейных дифференциальных уравнений, указанных в таблице: (11) и (17) размерности $\left(d_{2}+1\right) \times\left(d_{2}+1\right)$ и $(13)$ и $(16)$ размерности $\left(d_{1}+1\right) \times\left(d_{1}+1\right)$ в совокупности с лестничными соотношениями (10), (12), (14), (15). Мы не рассматривали деформационные уравнения, т.е. дифференциальные уравнения, получаемые при инфинитезимальных вариациях коэффициентов потенциалов $V_{1}$ и $V_{2}$, входящих в меру интегрирования. Полное исследование этих деформаций содержится в работе [8]. В частности, в этой работе показано, что получаемая при этом переопределенная система уравнений с частными производными оказывается совместной. Заметим, что смешанная система ОДУ и разностных уравнений также оказывается совместной, на что указывает следующее предложение.

\begin{tabular}{|l|l|}
\hline Размер $\left(d_{2}+1\right) \times\left(d_{2}+1\right)$ & Размер $\left(d_{1}+1\right) \times\left(d_{1}+1\right)$ \\
\hline$-\frac{1}{K} \frac{d}{d x} \Psi(x)=\stackrel{N}{D}_{1}(x) \underset{N}{\Psi}(x)$ & $\frac{1}{K} \frac{d}{d y} \underline{\Psi}^{N-1}(y)=\underline{\Psi}^{N-1}(y) \underline{D}_{2}(y)$ \\
$\frac{1}{K} \frac{d}{d x} \underline{N} \underline{\Phi}(x)=\underline{N}^{N-1}(x) \underline{N}_{1}(x)$ & $-\frac{1}{K} \frac{d}{d y} \Phi_{N}(y)=\stackrel{D}{2}_{2}(y) \Phi_{N}(y)$ \\
\hline
\end{tabular}

ПРЕДЛОЖЕНИЕ 2. Лестничные матрицы $\underset{N}{\mathbf{a}}$ перемешивают дифференииальные системы $D_{1}$ с различными $N$, т.е.

$$
\underset{N}{\mathbf{a}}(x)\left(\frac{d}{d x}+\stackrel{N}{D}_{1}(x)\right)=\left(\frac{d}{d x}+\stackrel{N+1}{D}_{1}(x)\right) \underset{N}{\mathbf{a}}(x)
$$

Аналогичные утверждения верны для трех других последовательностей ОДУ и соответствующих лестничных соотношений.

Следуюшее предложение объясняет, как четыре последовательности систем (11), $(17),(13)$ и (16) связаны между собой с помощью спариваний Кристоффеля-Дарбу.

ПРЕДЛОЖЕНИЕ 3. Выполняются следующие соотношения:

$$
\begin{array}{ll}
\stackrel{N}{D}_{1}(x) \stackrel{N}{\mathbb{A}}=\stackrel{N}{\mathbb{A}} D_{1}(x), & \underline{N}_{2}(y) \stackrel{N}{\mathbb{B}}=\stackrel{N}{\mathbb{B}} \stackrel{N}{D}_{2}(y) .
\end{array}
$$


Спектры двух матриц $D_{1}(x)$ и $\underline{D}_{1}(x)$ (т.е. их характеристические многочлены) совпадают, равно как и спектры матриц $D_{2}(y)$ и $\underline{D}_{2}(y)$. Более того, имеет место и менее очевидная спектральная дуальность. В самом деле, в работе [8] было доказано, что

$$
\operatorname{det}\left(y \mathbf{1}-\stackrel{N}{D}_{1}(x)\right)=c \operatorname{det}\left(x \mathbf{1}-\stackrel{N}{D}{ }_{2}(y)\right),
$$

где $c$ - отношение старших коэффициентов потенциалов $V_{1}$ и $V_{2}$. Заметим, что эти два определителя содержат квадратные матрицы ранга $d_{2}+1$ в левой части и ранга $d_{1}+1$ в правой части. В следующем разделе будет дан простой вывод "наивного" предела $N \rightarrow \infty$ для этих результатов, а именно предела, в котором соответствуюшие матрицы рекурсии $P$ и $Q$ считаются коммутируюшими.

\section{3. АБЕЛЕВ СЛУЧАЙ}

В данном разделе выводится свойство спектральной дуальности в частном случае предела $N \rightarrow \infty, K / N=\mathcal{O}(1)$. В таком пределе матрицы $P$ и $Q$, при том что они сохраняют свою зонную структуру, могут рассматриваться как коммутируюшие, потому что $[P, Q]=-\mathbf{1} / K \rightarrow 0$. Кроме того, рассматривается только тот случай, когда коэффициенты $\alpha_{j}(n), \beta_{j}(n)$ и $g(n)$ не зависят от $n$. Это довольно сильное требование, которое выполнено только для определенного диапазона констант связи. Такой предел исследовался в литературе, где он получил название "одноразрезного случая" или "случая рода ноль" [1], [3].

Дальнейшее упрошение, носящее чисто технический характер, можно получить, если рассмотреть матрицы по-настоящему бесконечного (дважды бесконечного) размера, т.е. размера $\mathbb{Z} \times \mathbb{Z}$ вместо $\mathbb{N} \times \mathbb{N}$. Покажем, что свойство спектральной дуальности в этом случае сводится к классическому результату из коммутативной алгебры, а именно к вычислению результанта двух многочленов Лорана.

Неабелев случай (т.е. случай конечных $N$ ) был детально исследован в работе [8]. Подход, использованньй в этой работе, может быть также успешно применен к выводу результатов в пределе $N \rightarrow \infty$. Тем не менее в данной работе мы приводим другое доказательство, и это доказательство также может быть применено к неабелеву случаю [9].

Уравнения $[P, Q]=-(1 / K) \mathbf{1}$ в пределе $N \rightarrow \infty, K=\mathcal{O}(N)$, становятся уравнениями коммутативности $[P, Q]=0$. Более того, поскольку рассматриваются конечнозонные матрицы и основное внимание уделяется окну при размере матрицы $N$, можно заменить полубесконечные матрицы $P, Q$ дважды бесконечными матрицами с той же зонной структурой. При подходяших масштабных преобразованиях из физических соображений следует, что последовательности $\gamma(n), \alpha_{j}(n)$ и $\beta_{k}(n)$ в действительности не зависят от $n($ если $n=\mathcal{O}(N))$. Именно этот простой случай мы и хотим рассмотреть в данной работе.

Две коммутирующие матрицы $P$ и $Q$ с одной и той же зонной структурой теперь становятся просто многочленами от матрицы сдвига. Все матрицы предполагаются матрицами размера $\mathbb{Z} \times \mathbb{Z}$, и, следовательно, матрица $\Lambda=\left[\delta_{i, i+1}\right]$ оказывается обратимой, а ее 
обратная - это просто транспонированная матрица $\Lambda^{\mathrm{t}}$. С учетом этого можно записать

$$
\begin{array}{ll}
Q(\Lambda):=\gamma \Lambda+\alpha_{0}+\sum_{i=1}^{d_{2}} \alpha_{i} \Lambda^{-i}, & \gamma \neq 0, \quad \alpha_{d_{2}} \neq 0, \\
P(\Lambda):=\gamma \Lambda^{-1}+\beta_{0}+\sum_{i=1}^{d_{1}} \beta_{i} \Lambda^{i}, & \gamma \neq 0, \quad \beta_{d_{1}} \neq 0,
\end{array}
$$

где $Q$ и $P$ рассматриваются как многочлены Лорана от переменных $\Lambda, \Lambda^{-1}$. Удобно ввести переменную $\lambda$ и выразить многочлены $Q$ и $P$ посредством их действия на градуированном пространстве:

$$
Q, P: \mathbb{C}\left[\lambda, \lambda^{-1}\right] \rightarrow \mathbb{C}\left[\lambda, \lambda^{-1}\right],
$$

задаваемого соотношениями (18) при замене $\Lambda$ на $\lambda$. Матрица сдвига $\Lambda$ задается обычным умножением на $\lambda$, в то время как матрица $\Lambda^{\mathrm{t}}=\Lambda^{-1}$ представляется умножением на переменную $\lambda^{-1}$. Эквивалентом окна при этом является линейная оболочка, образованная $d_{2}+1$ последовательными мономами от переменной $\lambda$ :

$$
\mathbb{C}\left\{\psi_{N-d_{2}}, \ldots, \psi_{N}\right\} \leftrightarrow \mathbb{C}\left\{\lambda^{N-d_{2}}, \ldots, \lambda^{N}\right\}
$$

Процедура складьвания в этом случае сводится к весьма простому выражению. В самом деле, складывание градуированного пространства $W:=\mathbb{C}[[\lambda]]$ на линейную оболочку векторов $\lambda^{N-d_{2}}, \ldots, \lambda^{N}$ сводится просто к взятию частного

$$
\mathbb{C}[[\lambda]] \simeq \mathbb{C}[x] \otimes \mathbb{C}[[\lambda]] \quad(\bmod x-Q(\lambda)=0) \simeq \mathbb{C}[x]\left\{\lambda^{N-d_{2}}, \ldots, \lambda^{N}\right\} .
$$

Другими словами, моном $\lambda^{N+1}$ может быть представлен в терминах мономов $\lambda^{N-d_{2}}, \ldots$ $\ldots, \lambda^{N}$ с помошью соотношения $x-Q(\lambda)=0$. Эквивалентом лестничной матрицы будет теперь просто операция умножения на $\lambda$ в "сложенном" окне $\mathbb{C}[x]\left\{\lambda^{N-d_{2}}, \ldots, \lambda^{N}\right\}$. Эта процедура определена таким образом, чтобы сделать коммутативной следующую диаграмму:

$$
\begin{array}{ccc}
\mathbb{C}[[\lambda]] & \mathbb{C}[[\lambda]] \\
\langle x-Q(\lambda)=0\rangle \downarrow & \downarrow\langle x-Q(\lambda)=0\rangle \\
\mathbb{C}[x]\left\{\lambda^{N-d_{2}}, \ldots, \lambda^{N}\right\} \stackrel{\mathbf{a}(x)}{\longrightarrow} \mathbb{C}[x]\left\{\lambda^{N-d_{2}}, \ldots, \lambda^{N}\right\} .
\end{array}
$$

В принципе величина а могла бы зависеть от $N$, но легко видеть, что она задается следуюшей похожей на (14) матрищей, не завислшей от $N$ :

$$
\mathbf{a}(x)=\left[\begin{array}{ccccc}
0 & 1 & \ldots & \ldots & 0 \\
0 & 0 & \ddots & \ddots & \vdots \\
0 & 0 & \ddots & \ddots & 1 \\
-\frac{\alpha_{d_{2}}}{\gamma} & \ldots & \ldots & -\frac{\alpha_{1}}{\gamma} & \frac{x-\alpha_{0}}{\gamma}
\end{array}\right] .
$$


Аналогично можно было бы определить другое складывание вдоль направления $P$ с помошью следуюшей диаграммы:

$$
\begin{array}{ccc}
\mathbb{C}[[\lambda]] & \lambda & \mathbb{C}[[\lambda]] \\
\langle y-P(\lambda)=0\rangle \downarrow & & \downarrow\langle y-P(\lambda)=0\rangle \\
\mathbb{C}[y]\left\{\lambda^{N-1}, \ldots, \lambda^{N+d_{1}-1}\right\} & \stackrel{\mathbf{b}(y)}{\longleftarrow} & \mathbb{C}[y]\left\{\lambda^{N-1}, \ldots, \lambda^{N+d_{1}-1}\right\},
\end{array}
$$

где матрица $\mathbf{b}$ имеет вид

$$
\mathbf{b}(y)=\left[\begin{array}{ccccc}
0 & 1 & \ldots & \ldots & 0 \\
0 & 0 & \ddots & \ddots & \vdots \\
\ldots \ldots & \ldots & \ldots & \ldots \ldots & \ldots \\
0 & 0 & \ddots & \ddots & 1 \\
-\frac{\beta_{d_{1}}}{\gamma} & \ldots & \ldots & -\frac{\beta_{1}}{\gamma} & \frac{y-\beta_{0}}{\gamma}
\end{array}\right]
$$

В этом подходе матрицы $D_{1}(x)$ и $D_{2}(y)$ имеют следуюший простой вид:

$$
\begin{aligned}
& D_{1}(x):=P(\mathbf{a}(x))=\gamma \mathbf{a}(x)^{-1}+\sum_{j=0}^{d_{1}} \beta_{j} \mathbf{a}^{j}(x), \\
& D_{2}(y):=Q(\mathbf{b}(y))=\gamma \mathbf{b}(y)^{-1}+\sum_{j=0}^{d_{2}} \alpha_{j} \mathbf{b}^{j}(y) .
\end{aligned}
$$

Прежнее утверждение относительно спектральной дуальности теперь сводится к тождеству

$$
\operatorname{det}\left(y \mathbf{1}-D_{1}(x)\right) \propto \operatorname{det}\left(x \mathbf{1}-D_{2}(y)\right) .
$$

Ниже будет показано, что оба эти определителя на самом деле представляют собой результанты (относительно переменной $\lambda$ ) двух многочленов Лорана $Q(\lambda)-x$ и $P(\lambda)-y$. Доказательство этого для многочленов вполне стандартно и требует лишш простой адаптации к случаю многочленов Лорана (см., например, [11]). Это доказательство сводится к исследованию следующего вложения:

$$
\begin{aligned}
& \mathbb{C}\left\{\lambda^{N-d_{2}}, \ldots, \lambda^{N}\right\} \oplus \\
& \quad \oplus \mathbb{C}\left\{\lambda^{N-1}, \ldots, \lambda^{N+d_{1}-1}\right\} \stackrel{(P(\lambda)-y) \oplus(Q(\lambda)-x)}{\longrightarrow} \mathbb{C}\left\{\lambda^{N-d_{2}-1}, \ldots, \lambda^{N+d_{1}}\right\},
\end{aligned}
$$

где $x$ и $y$ рассматриваются как параметры вложения. Обозначим через $W, \underline{W}$ и $U$ три векторных пространства:

$$
\begin{gathered}
W:=\mathbb{C}\left\{\lambda^{N-d_{2}}, \ldots, \lambda^{N}\right\}, \quad \underline{W}:=\mathbb{C}\left\{\lambda^{N-1}, \ldots, \lambda^{N+d_{1}-1}\right\}, \\
U:=\mathbb{C}\left\{\lambda^{N-d_{2}-1}, \ldots, \lambda^{N+d_{1}}\right\} .
\end{gathered}
$$


Тогда рассматриваемое вложение можно собрать в одно отображение

$$
W \oplus \underline{W} \stackrel{(P(\lambda)-y) \oplus(Q(\lambda)-x)}{\longrightarrow} U
$$

Две части этого отображения дают пространства, которые оказываются перпендикулярными в случае обшего положения при изменении переменных $x$ и $y$. Если они не перпендикулярны для данной пары значений $(x, y)$, то это значит, что сушествуют $w \in W$ и $\underline{w} \in \underline{W}$ такие, что

$$
w \neq 0, \quad \underline{w} \neq 0, \quad(P-y) w=(Q-x) \underline{w} \in U .
$$

Вычисляя частное от деления этого соотношения на соотношение $Q(\lambda)-x=0$ или $P(\lambda)-y=0$, получаем

$$
\begin{aligned}
& \left(D_{1}(x)-y\right) w=0, \\
& \left(D_{2}(y)-x\right) \underline{w}=0,
\end{aligned}
$$

откуда следует, что $y$ - собственное значение матрицы $D_{1}(x)$, а $x$ - собственное значение матрицы $D_{2}(y)$. Обратно, если выполнено какое-либо одно из уравнений $(19)$, например первое для ненулевого вектора $w$, то это значит, что сушествует $\underline{w} \in \underline{W}$ такое, что

$$
(P-y) w=(Q-x) \underline{w} .
$$

Заметим, что величина $(Q-x) \underline{w}$ не может быть равна нулю, поскольку отображение $P-y: W \rightarrow U$ инъективно при всех $y$, а потому $(P-y) w \neq 0$. Тот же результат получится, если мы начнем рассмотрение со второго уравнения (19). Тем самым это доказывает, что вложение не перпендикулярно тогда и только тогда, когда $x$ есть собственное значение оператора $D_{2}(y)$, что эквивалентно условию, что $y$ есть собственное значение оператора $D_{1}(x)$.

Условие поперечности сводится к тому, что определитель вложения не равен нулю (в любом фиксированном базисе). Легко видеть, что такое вложение задается матрицей Сильвестра двух многочленов Лорана

$\left[\begin{array}{ccccccccc}\gamma & \beta_{0}-y & \beta_{1} & \ldots & \ldots & \beta_{d_{1}} & 0 & 0 & 0 \\ 0 & \gamma & \beta_{0}-y & \beta_{1} & \ldots & \ldots & \beta_{d_{1}} & 0 & 0 \\ 0 & 0 & \gamma & \beta_{0}-y & \beta_{1} & \ldots & \ldots & \beta_{d_{1}} & 0 \\ 0 & 0 & 0 & \gamma & \beta_{0}-y & \beta_{1} & \ldots & \ldots & \beta_{d_{1}} \\ \hline \alpha_{d_{2}} & \ldots & \alpha_{1} & \alpha_{0}-x & \gamma & 0 & 0 & 0 & 0 \\ 0 & \alpha_{d_{2}} & \ldots & \alpha_{1} & \alpha_{0}-x & \gamma & 0 & 0 & 0 \\ 0 & 0 & \alpha_{d_{2}} & \ldots & \alpha_{1} & \alpha_{0}-x & \gamma & 0 & 0 \\ 0 & 0 & 0 & \alpha_{d_{2}} & \ldots & \alpha_{1} & \alpha_{0}-x & \gamma & 0 \\ 0 & 0 & 0 & 0 & \alpha_{d_{2}} & \ldots & \alpha_{1} & \alpha_{0}-x & \gamma\end{array}\right]$,


определитель $\Delta(x, y)$ которой равен результанту. Простой подсчет степеней свободы и исследование старших степеней по переменным $x$ или $y$ показывают, что

$$
\alpha_{d_{2}} \gamma^{d_{1}} \operatorname{det}\left(y \mathbf{1}-D_{1}(x)\right)=\Delta(x, y)=\beta_{d_{1}} \gamma^{d_{2}} \operatorname{det}\left(x \mathbf{1}-D_{2}(y)\right)
$$

что задает спектральные кривые как местоположение непоперечных вложений. Пересечение двух вложений на этой спектральной кривой обычно задается одномерной кривой и проецируется на собственные векторы операторов $D_{1}(x)$ и $D_{2}(y)$.

В то время как это весьма простое рассуждение представляет собой просто переформулировку стандартных алгебраических результатов в нашем абелевом подходе, очень похожий подход может также быть использован при доказательстве спектральной дуальности для пары $\stackrel{N}{D}{ }_{1}(x)$ и $\underline{N}_{2}(y)$ в подходе с конечным размером матриц $N$, в котором матрицы $P$ и $Q$ не коммутируют. Улучшая и развивая этот подход, в работе [9] мы получили и другие результаты работы [8] в более элегантном и компактном виде. Среди этих результатов отметим условие согласования для уравнений деформаций констант связи в потенциалах $V_{1}, V_{2}$, что, в частности, влечет инвариантность обобшенных монодромий операторов $\partial_{x}+\stackrel{N}{D}{ }_{1}(x)$ и $\partial_{y}+\stackrel{N}{D}{ }_{2}(y)$. При этом получаются некоммутативные аналоги результантов для конечнозонных матриш, свойства которых будут исследованы в дальнейшем.

\section{Список литературы}

[1] J. M. Daul, V. Kazakov, I. K. Kostov. Nucl. Phys. B. 1993. V. 409. P. 311; hep-th/9303093.

[2] N. M. Ercolani, K. T.-R. McLaughlin. Physica D. 2001. V. 152-153. P. 232.

[3] B. Eynard. Nucl. Phys. B. 1997. V. 506. P. 633; cond-mat/9707005.

[4] B. Eynard. J. Phys. A. 1998. V. 31. P. 8081; cond-mat $/ 9801075$.

[5] B. Eynard, M. L. Mehta. J. Phys. A. 1998. V. 31. P. 4449; cond-mat/9710230.

[6] K. Ueno, K. Takasaki. Adv. Stud. Pure Math. 1984. V. 4. P. 1.

[7] M. Adler, P. Van Moerbeke. Commun. Pure Appl. Math. 1997. V. 50. P. 241; Ann. Math. 1999. V. 149. P. 921.

[8] M. Bertola, B. Eynard, J. Harnad. Commun. Math. Phys. 2002. V. 229. P. 73; nlin.SI/0108049.

[9] M. Bertola, B. Eynard, J. Harnad, J. Hurtubise. The geometric meaning of dual isomonodromy tau functions appearing in 2-matrix models. (in preparation).

[10] A. Userkesm, S. P. Norset. Constr. Approx. 1989. V. 5. P. 437.

[11] I. M. Gelfand, M. M. Kapranov, A. V. Zelevinsky. Discriminants, Resultants and Multidimensional Determinants. Boston, MA: Birkhäuser, 1994. 